\title{
A Universal Formula for Asymptotic Stabilization with Bounded Controls
}

\author{
Muhammad Nizam Kamarudin ${ }^{\mathrm{a}}$, Abdul Rashid Husain ${ }^{\mathrm{b}}$, Mohamad Noh Ahmad ${ }^{\mathrm{c}}$, Zaharuddin \\ Mohamed $^{\mathrm{d}}$ \\ ${ }^{a}$ Faculty of Electrical Engineering, Universiti Teknikal Malaysia Melaka (UTeM), Hang Tuah Jaya, \\ 76100 Durian Tunggal, Melaka, Malaysia. \\ ${ }_{\mathrm{b}, \mathrm{c}, \mathrm{d}}$ Faculty of Electrical Engineering, Universiti Teknologi Malaysia, UTM Skudai,81310 Johor, Malaysia.
}

\begin{tabular}{|c|c|}
\hline Article Info & ABSTRACT \\
\hline Article history: & Motivated by Artstein and Sontag universal formula, this brief paper presents \\
\hline Received Oct 14, 2014 & $\begin{array}{l}\text { an explicit proof of the universal formula for asymptotic stabilization and } \\
\text { asymptotic disturbance rejection of a nonlinear system with mismatched }\end{array}$ \\
\hline Revised Dec 19, 2014 & uncertainties and time varying disturbances. We prove the stability via \\
\hline Accepted Jan 7, 2015 & $\begin{array}{l}\text { Lyapunov stability criteria. We also prove that the control law satisfies small } \\
\text { control property such that the magnitude of the control signal can be bounded }\end{array}$ \\
\hline Keyword: & $\begin{array}{l}\text { without the catastropic effect to the closed loop stability. For clarity, we } \\
\text { benchmark the proposed approach with other method namely a Lyapunov }\end{array}$ \\
\hline $\begin{array}{l}\text { Bounded controls } \\
\text { Lyapunov stability }\end{array}$ & $\begin{array}{l}\text { redesign with nonlinear damping function. We give a numerical example to } \\
\text { verify the results. }\end{array}$ \\
\hline
\end{tabular}

Copyright $(2015$ Institute of Advanced Engineering and Science. All rights reserved.

\section{Corresponding Author:}

Muhammad Nizam Kamarudin

Faculty of Electrical Engineering

Universiti Teknikal Malaysia Melaka (UTeM), 76100 Durian Tunggal, Melaka, MALAYSIA

Tel: +6016-2022457 / Fax: +606-5552222

E-mail: nizamkamarudin@utem.edu.my

\section{INTRODUCTION}

Stabilizing systems with uncertainty and exogenous disturbance requires massive control energy. For illustration, it is easy to stabilize unstable system by forcing their poles to the left-hand-side of the Splane so that the closed-loop system stable. Theoretically, placing the closed-loop poles near to $-\infty$ render fast convergence rate but require high energy as trade-off. In some industrial cases are DC drive systems where the constraints are due to the physical limitation of the motor drive such as converter protection, magnetic saturation and motor overheating that make the current command limited to an admissible set of input. For another case such as electric vehicles where the controlled variable is a speed, the motor torque or voltage may be bounded within allowable ranges.

There are various attempts to stabilize nonlinear system with mismatched uncertainty and time varying disturbance. Such attempts have been addressed in [1]-[5]. In [1], a nonlinear damping function is augmented to the nominal controller during the Lyapunov redesign phase. In this paper, we begin with normal feedback control law by using a Lyapunov redesign techniqu. During Lyapunov redesign phase, a nonlinear damping function is used to combat with uncertainty or exogenous disturbance. By means of comparing square, we then improve the control law complexity by avoiding the cancellation of a useful nonlinear term. Lastly, we introduce a "universal-like" formula to stabilize the system with less control effort. As such, main objective of this paper is to provide an improved universal formula due to Artstein [6] and Sontag [7-8], such that it can be applied to nonlinear systems with mismatched uncertainties and time varying 
disturbances. The introduced formula is generalized, simple, explicit, and in a sense of "universal", in order to obtain global asymptotic stability and global disturbance rejection with less control effort.

Let concern single-input-single-output, one-dimensional nonlinear system of the form:

$$
\dot{x}=f(x)+g(x) u+h(x, t)
$$

with state $x(t) \in \mathbb{R}$ and control $u \in \mathbb{R} . f(x)$ and $g(x)$ are analytic, smooth vector fields, which are infinitely differentiable. A smooth function $h(x, t)$ represents the sum of uncertainties and exogenous disturbances. For feedback stabilization, the existence of a control-Lyapunov function is necessary, as in Artstein's theorem:

"Artstein theorem states that a dynamical system has a differentiable control-Lyapunov function if and only if there exists a regular stabilizing feedback"

-Artstein [6]-

Therefore, there exists a smooth, proper and positive definite control-Lyapunov function $V: \mathbb{R}$ for the system in equation (1) where conditions $V(0)=0, V(x)>0$ for $x \neq 0$, and $V(x) \rightarrow \infty$ as $\|x\| \rightarrow \infty$ are valid. Recall from [8] that the existence of such a control-Lyapunov function implies that the system is asymptotically controllable provided that the derivative of $V: \mathbb{R}^{n}$ negative definite. As such, there must be a feedback control law:

$$
u=K(x)-\frac{-f(x) h^{2}(x, t)}{g(x) f(x) h(x, t)+\tau e^{-\alpha t}}, K>0
$$

which globally stabilize the system in equation (1). Note that, for stability, high control magnitude $u \in \mathbb{R}^{m}$ is required in order to push systems poles to the left hand side of the s-plane. Thus, the regular feedback law in equation (2) is unbounded in magnitude, as well as high in energy consumption. Our control problem now is to limit $u \in \mathbb{R}^{m}$ within $-u_{\text {lower }}<u<u_{\text {upper }}$ such that the closed loop system

$$
\dot{x}=f(x)+g(x) K(x)
$$

remain Hurwitz and $h(x, t)$ perished as $t \rightarrow \infty$ in order to preserve a global disturbance rejection.

\section{METHODOLOGY - UNIVERSAL FORMULA FOR ROBUST BOUNDED CONTROL}

Consider nonlinear system in equation (1) and a control-Lyapunov function $V: \mathbb{R}$. There exist operators $F \in \mathbb{R}, G_{1} \in \mathbb{R}, G_{2} \in \mathbb{R}$, and $G_{3} \in \mathbb{R}$, where:

$$
\begin{aligned}
& F=\left(\frac{\partial V}{\partial x}\right)^{T} \cdot f(x) \\
& G_{1}=\left(\frac{\partial V}{\partial x}\right)^{T} \cdot g(x) \\
& G_{2}=x \cdot g(x) \\
& G_{3}=\frac{h(x, t)}{g(x)}
\end{aligned}
$$

There also exist a scalar $\tau>0$ and $\alpha>0$, such that the robust bounded control law:

$$
u\left(F, G_{1}, t\right)=-\frac{K\left(F+\sqrt{F^{2}+G_{1}^{4}}\right)}{G_{1}\left(1+\sqrt{1+G_{1}^{2}}\right)}-\frac{G_{2} G_{3}^{2}}{G_{2} G_{3}+\tau e^{-\alpha t}}, K>0
$$

satisfies small control property for the system in equation (1) (see definition 1) and also guarantees the asymptotic stability and the asymptotic disturbance rejection (which means robust toward $h(x, t)$ ). 
Definition 1: Small Control Property

For the system in equation (1) satisfies small control property, there is a known control-Lyapunov function $V: \mathbb{R}$. For every $\varepsilon>0$, there exists a $\delta(\varepsilon)>0$ so that for all $x \neq 0$ and $\|x\|<\delta$, there is control $\left\|u\left(F, G_{1}, t\right)\right\|<\varepsilon$ such that $\dot{x}=f(x)+g(x) u\left(F, G_{1}, t\right)<0$.

In what follows, Lemma 1 is useful to reach the stability proof of the robust bounded control law in equation (8).

Lemma 1 [1]:

Assume that $F, G_{1}$ in equation (8) are real numbers. And there exists real number $\varepsilon$ such that $F<\varepsilon\left|G_{1}\right|$, and $0<\varepsilon \leq \varphi$ for $\varphi \in \mathbb{R}$. Therefore, there exists a nominal stabilizing function $u\left(F, G_{1}\right)=-\frac{K\left(F+\sqrt{F^{2}+G_{1}^{4}}\right)}{G_{1}\left(1+\sqrt{1+G_{1}^{2}}\right)}$ with property:

$$
\left|u\left(F, G_{1}\right)\right|<\min \left\{2 \varepsilon+\left|G_{1}\right|, \varepsilon\right\}
$$

Proof of Lemma 1

If $G_{1}=0$, then the solution for $u\left(F, G_{1}\right)$ is trivial. Then, we assume that $G_{1} \neq 0$. Since $\left|u\left(F, G_{1}\right)\right|=$ $\left|u\left(F,-G_{1}\right)\right|$, then $G_{1}>0$ and $F<\varepsilon G_{1}$. If $F>0$, then $F<\varepsilon\left|G_{1}\right|$ and $0<\varepsilon \leq 1$. With control parameter $K=1$, we can see that nominal stabilizing function $\left|u\left(F, G_{1}\right)\right|$ bounded by $\varphi$ as $\varepsilon \leq \varphi$, and also bounded by its numerator. This yield:

$$
\begin{aligned}
u\left(F, G_{1}\right) & =\frac{F+\sqrt{F^{2}+G_{1}^{4}}}{G_{1}\left(1+\sqrt{1+G_{1}^{2}}\right)} \\
& =\frac{\varepsilon G_{1}+\sqrt{\left(\varepsilon G_{1}\right)^{2}+G_{1}^{4}}}{G_{1}\left(1+\sqrt{1+G_{1}^{2}}\right)} \\
& =\frac{\varepsilon+\sqrt{\varepsilon^{2}+G_{1}^{2}}}{1+\sqrt{1+G_{1}^{2}}} \\
& <\min \left\{2 \varepsilon+\left|G_{1}\right|, \varphi\right\}
\end{aligned}
$$

Proof of Stability:

By referring to Lemma 1, the proof of stability for the robust bounded control law in equation (8) is presented. With control-Lyapunov function $V=0.5 x^{2}: \mathbb{R}>0$, the derivative of $V: \mathbb{R}$ along $\mathrm{x}$ renders

$$
\begin{aligned}
& \dot{V}=x(f(x)+g(x) u+h(x, t)) \\
& =x\left(f(x)+g(x)\left(-\frac{K\left(F+\sqrt{F^{2}+G_{1}^{4}}\right)}{G_{1}\left(1+\sqrt{1+G_{1}^{2}}\right)}-\frac{G_{2} G_{3}^{2}}{G_{2} G_{3}+\tau e^{-\alpha t}}\right)+h(x, t)\right) \\
& =x\left(f(x)-g(x) \frac{K\left(F+\sqrt{F^{2}+G_{1}^{4}}\right)}{G_{1}\left(1+\sqrt{1+G_{1}^{2}}\right)}\right)+x\left(g(x) \frac{-G_{2} G_{3}^{2}}{G_{2} G_{3}+\tau e^{-\alpha t}}+h(x, t)\right) \\
& \leq x\left(-\frac{F+\sqrt{F^{2}+G_{1}^{4}}}{G_{1}\left(1+\sqrt{1+G_{1}^{2}}\right)} K x\right)+x g(x) \frac{-G_{2} G_{3}^{2}}{G_{2} G_{3}+\tau e^{-\alpha t}+x h(x, t)} \\
& \leq-\frac{F+\sqrt{F^{2}+G_{1}^{4}}}{G_{1}\left(1+\sqrt{1+G_{1}^{2}}\right)} K x^{2}+\frac{-\left\|G_{2} G_{3}\right\|^{2}}{G_{2} G_{3}+\tau e^{-\alpha t}}+G_{2} G_{3} \\
& \leq-\frac{F+\sqrt{F^{2}+G_{1}^{4}}}{G_{1}\left(1+\sqrt{1+G_{1}^{2}}\right)} K x^{2}+\frac{-\left\|G_{2} G_{3}\right\|^{2}+\left\|G_{2} G_{3}\right\|^{2}+G_{2} G_{3} \tau e^{-\alpha t}}{G_{2} G_{3}+\tau e^{-\alpha t}} \\
& \leq-\frac{F+\sqrt{F^{2}+G_{1}^{4}}}{G_{1}\left(1+\sqrt{1+G_{1}^{2}}\right)} K x^{2}+\tau e^{-\alpha t}
\end{aligned}
$$


With Lemma 1, we can further prove that:

$$
\dot{V} \leq-K\left(\min \left\{2 \varepsilon+\left|G_{1}\right|, \varphi\right\}\right) x^{2}+\tau e^{-\alpha t}
$$

This completes the proof for asymptotic stability. When $t \rightarrow \infty$, term $\tau e^{-\alpha t}$ vanishes to confirm the asymptotic disturbance rejection of the system in equation (1).

\section{NUMERICAL EXAMPLE}

Consider one-dimensional nonlinear system:

$$
\dot{x}=-x^{2}-x^{3}-u-h(x, t)
$$

with state $x(t) \in \mathbb{R}$ and control $u \in \mathbb{R}$. The uncertainty with time varying disturbance is denoted as

$$
h(x, t)=x^{2} \sin (x, t)
$$

In what follows, we stabilized the system in equation (13) such that the state $x(t) \in \mathbb{R}$ is asymptotically stable towards perturbed initial state $x(0)$ and also achieve the asymptotic disturbance rejection toward $h(x, t)$. We present two approaches; a Lyapunov redesign with nonlinear damping factor and the proposed bounded control in equation (8).

\subsection{Stabilization using Lyapunov Redesign and Nonlinear Damping Function}

Firstly, let stabilize system in equation (13) using direct Lyapunov technique with Lyapunov redesign and nonlinear damping function as addressed in [1]. Let consider system (13) in a form

$$
\dot{x}=f(x)+g(x)[u+\xi(x) \Delta(x, u, t)]
$$

where $\xi(x)$ is a vector of known smooth nonlinear function, and $\Delta(x, u, t)$ is the vector of uncertain nonlinearities and disturbance. Then, the control law renders the closed-loop system input-to-state stability for the system in equation (15) with respect to the disturbance input $\Delta(x, u, t)$. The function $d(x)$ is denoted as a stabilizing function for the nominal system in equation (15).

$$
u=d(x)-k \frac{\partial V}{\partial x}(x) g(x)|\xi(x)|^{2}, \quad k>0
$$

Likewise, for the system in equation (13), let $d_{1}(x) \equiv u$. We firstly seek $d_{1}(x)$ for the nominal unperturbed system in equation (13). Let there exists a control-Lyapunov function $V(x)=0.5 x^{2}$ such that the nominal stabilizing function in equation (17) renders the derivative of $V(x)$ along $x$ be a negative definite function (i.e. $\dot{V}(x) \leq-K_{1} x^{2}$ ).

$$
d_{1}(x)=x^{2}+x^{3}-K_{1} x \quad, \quad K_{1}>0
$$

Therefore, comparing equation (14) and equation (15) yields a vector of known smooth nonlinear function

$$
\xi(x)=x^{2}
$$

and a robust stabilizing function

$$
u_{1}=x^{2}+x^{3}-K_{1} x+K x \xi^{2}(x)
$$

\subsection{Cancellation Avoidance}

Control law in equation (19) is a straight forward design based on direct Lyapunov inspired by Artstein. Function $d_{1}(x)$ eliminates $x^{3}$-term in the system equation (13), which is known as a useful nonlinear term. As such, by using the comparing square, we devised a method to avoid the cancellation of a useful nonlinear term. Let again $d_{2}(x) \equiv u$, and recall a control-Lyapunov function $V(x) 0.5 x^{2}$. We then obtain its derivative along $\mathrm{x}$ as follows: 


$$
\begin{aligned}
\dot{V}(x) & =x\left[-d_{2}(x)-x\left(x^{2}+x\right)\right] \\
& =-x d_{2}(x)+\frac{1}{4} x^{2}-\left(x+\frac{1}{2}\right)^{2} x^{2}
\end{aligned}
$$

With that, $d_{1}(x)$ term in equation (17) can be reduced to

$$
d_{2}(x)=0.25 x+K_{1} x
$$

and hence reducing the control complexity of equation (19), as follows:

$$
u_{2}=\frac{1}{4} x+K_{1} x+K x \xi^{2}(x)
$$

By substituting equation (21) into equation (20), the asymptotic stability can be guaranteed via the derivative of $V(x)$, as follows:

$$
\dot{V}(x) \leq-K_{1} x^{2}-\left(x+\frac{1}{2}\right)^{2} x^{2}
$$

\subsection{Robust Bounded Control using Universal Formula}

This subsection is devoted to the application of the proposed universal formula in equation (8) to the numerical system in equation (13). With the control-Lyapunov function $V(x)=0.5 x^{2}$, we know from the standard form in equation (1) that:

$$
\left.\begin{array}{c}
\frac{\partial V}{\partial x}=x \\
f(x)=-x^{2}-x^{3} \\
g(x)=-1 \\
F=-x^{3}-x^{4} \\
G_{1}=-x \\
G_{2}=-x \\
G_{3}=-h(x, t)
\end{array}\right\}
$$

Therefore, the robust bounded control law is obtained as:

$$
u=-\frac{-x^{3}-x^{4}+\sqrt{x^{4}\left(x^{2}+2 x^{3}+x^{4}+1\right)}}{x\left(1+\sqrt{1+x^{2}}\right)} K_{1}-\frac{-x h^{2}(x, t)}{x h(x, t)+\tau e^{-\alpha t}}
$$

for all $K_{1}>0, \tau>0$ and $\alpha>0$.

\section{RESULTS AND ANALYSIS}

This section depicts simulation results for system in equation (13) by using a normal feedback control plus nonlinear damping function in equation (22) and a proposed bounded control law in equation (25). Figure 1 shows a stabilized $x$ for the pertubation of initial state $x(0)=\left[\begin{array}{ll}1 & 1\end{array}\right]^{T}$. Figure 2 shows the comparison in control signal. Figure 3 shows how the control signal react due to variation of the system state, $x$. We can observe that the signal produced by a bounded control law is confined at $u<\varepsilon$, hence satisfies small control property in definition 1. 


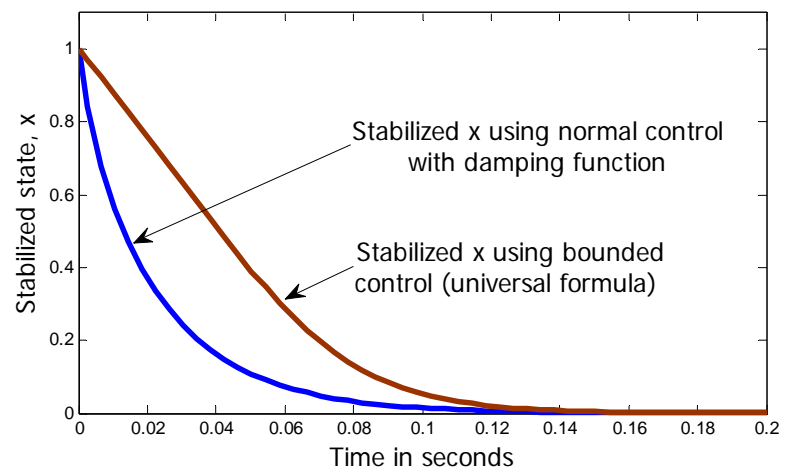

Figure 1. Stabilized state using control law in equation (22) and equation (25)

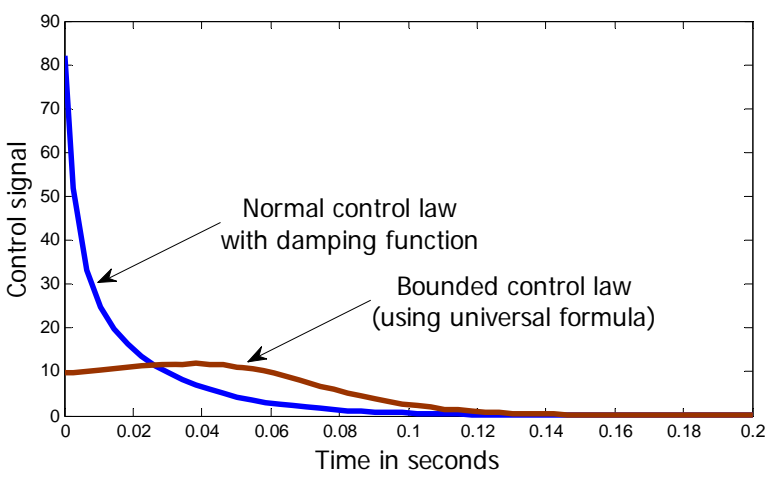

Figure 2. Control signal for control law in equation (22) and equation (25)

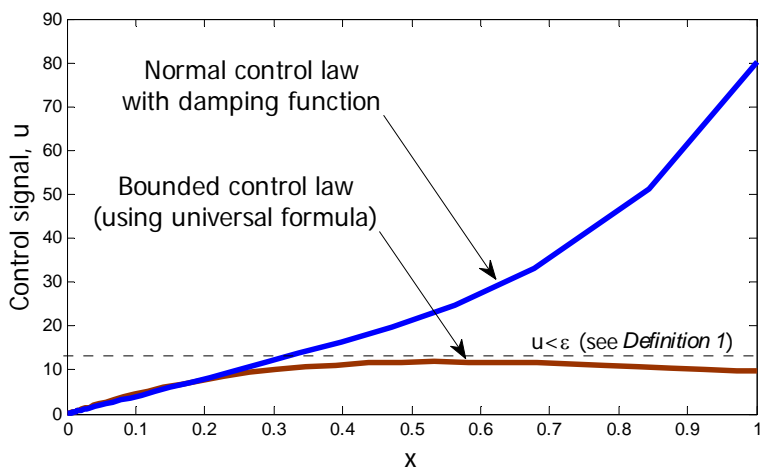

Figure 3. Control signal trajectories versus system trajectories

The control signals in Figure 2 are nonperiodic. Their magnitude and convergence rate are highly depend on how far the perturbed initial state $x(0)$ from the equilibria. To analyse the signal quantitatively, we compute the average power and energy by using Euler's approximation (see Appendix). For the perturbation in $x(0)=\left[\begin{array}{ll}1 & 1\end{array}\right]^{T}$, bounded control law require only 2,340.4 Joule of energy to steer $x(0)$ toward origin. While the normal control require 12,101 Joule of energy (see Table 1).

Table 1. Average energy produced by all control laws

\begin{tabular}{ccc}
\hline Control law & $\begin{array}{c}\text { Stabilizing } \\
\text { energy (Joule) }\end{array}$ & $\begin{array}{c}\text { Diminution } \\
\text { (percentage) }\end{array}$ \\
\hline Equation (22) & 12,101 & $80.66 \%$ \\
Equation (25) & $2,340.4$ & $8.4 \%$ \\
\hline
\end{tabular}




\section{CONCLUSION}

In this paper, we present a universal formula for bounded controller which is robust toward uncertainty and disturbance. The proposed method is universal for the system which affine in control. The appealing feature of the proposed controller is that, it is fairly easy to construct, guarantee the asymptotic stability and the asymptotic disturbance rejection, as well as bounded in the control signal magnitude. The numerical example and the simulation in this paper confirm the results.

\section{APPENDIX}

For a continuous signal in Figure 4, we can compute the average energy and power by using Euler's approximation. The average energy for a continuous signal shown in Figure 4:

$$
E_{u}(t)=\int_{-\infty}^{\infty}\|u(t)\|^{2} d t
$$

The average power for a continuous signal in Figure 4:

$$
\int_{a}^{b} u(t) d t \cong \sum_{n=0}^{N-1} u(a+n \Delta t) \Delta t
$$

where $N$ is the number of integral part in Euler's approximation, $b-a$ is the control signal duration and $\Delta t=(b-a) / N$ is the duration (or interval) for each integral part in seconds.

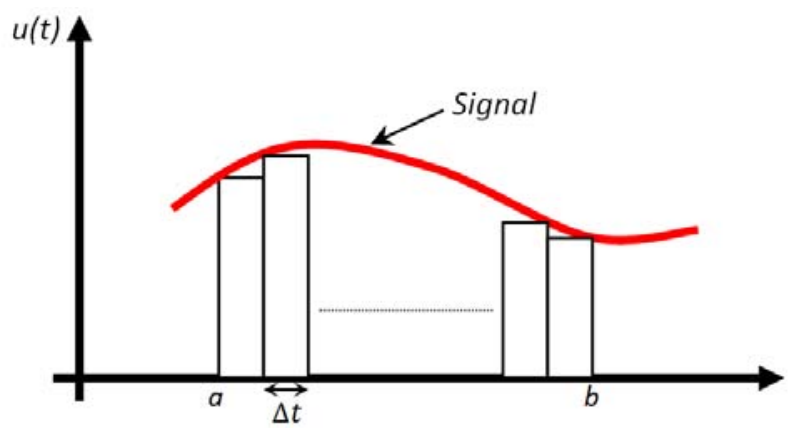

Figure 4. Euler’s approximation

\section{ACKNOWLEDGEMENTS}

We acknowledge the Ministry of Education Malaysia, Universiti Teknikal Malaysia Melaka (UTeM) and the Universiti Teknologi Malaysia (UTM) for research facilities and research collaboration.

\section{REFERENCES}

[1] M.N. Kamarudin, A.R. Husain and M.N. Ahmad, "Control of Uncertain Nonlinear Systems using Mixed Nonlinear Damping Function and Backstepping Techniques", IEEE International Conference on Control System, Computing and Engineering, pp. 105-109, 2012.

[2] M.T. Ravichandran and A.D. Mahindrakar, "Robust Stabilization of a Class of Underactuated Mechanical Systems Using Time Scaling and Lyapunov Redesign", IEEE Transactions on Industrial Electronics, vol. 58, pp. 42994313, 2011.

[3] M.N. Kamarudin, A.R. Husain and M.N. Ahmad, "Stabilization of uncertain systems using backstepping and Lyapunov redesign", The $4^{\text {th }}$ International Graduate Conference on Engineering Science \& Humanity, Johor, Malaysia, 2013.

[4] H.H. Choi, "An explicit formula of linear sliding surfaces for a class of uncertain dynamic systems with mismatched uncertainties", Automatica, vol. 34, pp. 1015-1020, 1998.

[5] H.H. Choi, "A new method for variable structure control system design: A linear matrix inequality approach", Automatica, vol. 33, pp. 2089-2092, 1997. 
[6] Z. Artstein, "Stabilization with relaxed controls", Nonlinear Analysis, Theory, Methods and Applications, vol. 7, pp. 1163-1173, 1983.

[7] L. Yuandan and E.D. Sontag, "A universal formula for stabilization with bounded controls", Systems \& Control Letters, vol. 16, pp. 393-397, 1991.

[8] E.D. Sontag, "A Universal Construction of Artstein Theorem on Nonlinear Stabilization", System and Control Letters, vol. 3, pp. 117-123, 1989.

\section{BIOGRAPHIES OF AUTHORS}

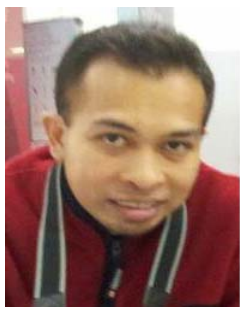

Muhammad Nizam Kamarudin was born in Selangor, Malaysia, in 1979. He received the B.Eng (Hons.) Electrical from the Universiti Teknologi MARA, Malaysian in 2002, and M.Sc Automation and Control from the University of Newcastle Upon Tyne, United Kingdom in 2007. He is currently with the Universiti Teknikal Malaysia Melaka (UTeM). He is the member of the Board of Engineers, Malaysia and Institute of Engineers, Malaysia. His research interests include nonlinear controls and robust control systems. Before joining UTeM, he worked as a technical engineer at the magnetron department of Samsung Electronics Malaysia.

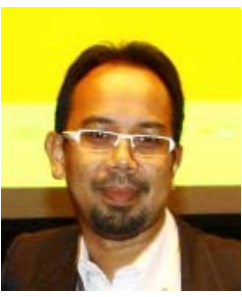

Abdul Rashid Husain received the B.Sc. degree in electrical and computer engineering from The Ohio State University, Columbus, Ohio, U.S.A, in 1997, M.Sc. degree in Mechatronics from University of Newcastle Upon Tyne, U.K., in 2003, and Ph.D. in Electrical Engineering (Control) from Universiti Teknologi Malaysia (UTM) in 2009. Before joining UTM, he worked as an engineer in semiconductor industry for several years specializing in precision molding and IC trimming process. He has taught courses in introduction to electrical engineering, microcontroller based system, modeling and control, and real-time control system. His research interests include application of control in dynamic system, network control system, real-time control system, and system with delay.

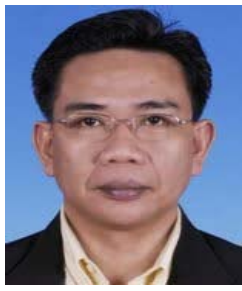

Mohamad Noh Ahmad received the Bachelor degree in Electrical Engineering from Universiti Teknologi Malaysia in 1986, the M.Sc. degree in Control Engineering from University of Sheffield, U.K. in 1988, and Ph.D. degree in Robotics from Universiti Teknologi Malaysia in 2003. Currently he is Associate Professor with the Department of Control and Mechatronic Engineering, Faculty of Electrical Engineering, Universiti Teknologi Malaysia. Since joining Universiti Teknologi Malaysia, his primary responsibilities include research and teaching in Robotics and Control Engineering. His researches involve among others modeling and control of numerous plants such as Active Magnetic Bearing System, Balancing Robot, and Direct-Drive Robot Manipulator.

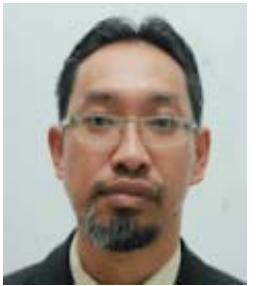

Zaharuddin Mohamed is an Associate Professor at the Department of Control and Mechatronics Engineering, Faculty of Electrical Engineering, Universiti Teknologi Malaysia. He received B.Eng in Electrical and Electronics Engineering from National University of Malaysia in 1993, M.Sc and Ph.D. in Control Systems Engineering from the University of Sheffield, UK in 1995 and 2003 respectively. He was a recipient of Islamic Development Bank (IDB) Merit Scholarship for his Ph.D. study. His research interests include command shaping control of dynamic systems, control of flexible structures and mechatronic systems. 